\title{
Pyruvate dehydrogenase E1 $\alpha$ represents a reliable prognostic predictor for patients with non-small cell lung cancer resected via curative operation
}

\author{
Ryuichi Ito ${ }^{1}$, Masakazu Yashiro ${ }^{2}$, Takuma Tsukioka ${ }^{1}$, Nobuhiro Izumi ${ }^{1}$, Hiroaki Komatsu ${ }^{1}$, \\ Hidetoshi Inoue $^{1}$, Yurie Yamamoto ${ }^{2}$, Noritoshi Nishiyama ${ }^{1}$ \\ ${ }^{1}$ Department of Thoracic Surgery, Osaka City University, Osaka, Japan; ${ }^{2}$ Molecular Oncology and Therapeutics, Osaka City University Graduate \\ School of Medicine, Osaka, Japan \\ Contributions: (I) Conception and design: R Ito, M Yashiro; (II) Administrative support: R Ito, M Yashiro; (III) Provision of study materials or \\ patients: R Ito, T Tsukioka, N Izumi, H Komatsu, N Nishiyama; (IV) Collection and assembly of data: R Ito, Y Yamamoto; (V) Data analysis and \\ interpretation: R Ito, T Tsukioka, N Izumi, H Komatsu, N Nishiyama; (VI) Manuscript writing: All authors; (VII) Final approval of manuscript: All \\ authors. \\ Correspondence to: Masakazu Yashiro, MD. Molecular Oncology and Therapeutics, Osaka City University Graduate School of Medicine, 1-4-3 \\ Asahimachi, Abeno-ku, Osaka 545-8585, Japan. Email: m9312510@med.osaka-cu.ac.jp.
}

Background: Lung cancer is associated with a high morbidity and mortality rate worldwide; however, no reliable and independent prognostic predictor for non-small cell lung cancer (NSCLC) after curative surgery is available. Glucose metabolism is correlated with cancer cell proliferation. Pyruvate dehydrogenase E1 $\alpha$ (PDH-E1 $\alpha$ ) catalyzes the conversion of pyruvate to acetyl-CoA and promotes aerobic glucose metabolism. In this study, we examined the relationship between PDH-E1 $\alpha$ expression and clinicopathological factors associated with NSCLC to identify a reliable prognostic predictor of NSCLC after curative surgery.

Methods: A total of 445 patients with NSCLC who underwent curative resection were enrolled in this study. PDH-E1 $\alpha$ expression was evaluated via immunohistochemistry. We analyzed the correlation between PDH-E1 $\alpha$ expression and clinicopathological features of the patients.

Results: In total, 248 (56\%) of the 445 patients with NSCLC were PDH-E1 $\alpha$-positive, and 197 patients were $\mathrm{PDH}-\mathrm{E} 1 \alpha$-negative. PDH-E1 $\alpha$ positivity was significantly correlated with the presence of adenocarcinoma $(\mathrm{P}<0.001)$ compared to the $\mathrm{PDH}-\mathrm{E} 1 \alpha$-negative group. Patients with NSCLC showing $\mathrm{PDH}-\mathrm{E} 1 \alpha$-negative expression had a significantly poorer overall survival rate $(\mathrm{P}=0.007)$ than those showing PDH-E1 $\alpha$-positive expression, especially at stage II. Patients with PDH-E1 $\alpha$ negative expression also showed a poorer disease-free survival rate $(\mathrm{P}=0.02)$. Multivariate analysis revealed that $\mathrm{PDH}-\mathrm{E} 1 \alpha$ negativity $(\mathrm{P}=0.037)$ and male sex $(\mathrm{P}<0.001)$ were significantly correlated with a poor overall survival.

Conclusions: $\mathrm{PDH}-\mathrm{E} 1 \alpha$ may represent a reliable prognostic predictor for NSCLC in patients that have recently undergone curative resection, especially at stage II.

Keywords: Pyruvate dehydrogenase (PDH); non-small cell lung cancer (NSCLC); predictive marker; curative resection

Submitted Sep 06, 2021. Accepted for publication Sep 16, 2021.

doi: $10.21037 /$ jtd-21-1463

View this article at: https://dx.doi.org/10.21037/jtd-21-1463

(c) Journal of Thoracic Disease. All rights reserved. 


\section{Introduction}

Lung cancer has a high morbidity and mortality rate worldwide, and is associated with the poor prognosis despite recent advances in therapy (1). Non-small cell lung cancer (NSCLC) accounts for approximately $85 \%$ of all lung cancers. Several clinicopathological prognostic factors, such as pathological stage, mutations in genes, and smoking history, have been reported to be associated with the outcome after surgery in patients with $\operatorname{NSCLC~}(2,3)$; however, no reliable and independent prognostic predictor for NSCLC after curative surgery has been identified at present.

Glucose metabolism in cancer cells is increased considerably with increasing proliferation $(4,5)$. In particular, anaerobic glycolysis is dominant even under aerobic conditions in the tumor microenvironment, which is known as the Warburg effect (6), suggesting that the enzymes involved in glucose metabolism in cancer cells may represent an important indicator of cancer malignancy $(7,8)$. Among the glucose metabolism enzymes, the pyruvate dehydrogenase $(\mathrm{PDH})$ complex catalyzes the conversion of pyruvate to acetyl-CoA and promotes aerobic glucose metabolism; $\mathrm{PDH}$ is phosphorylated by PDH kinase (PDK). The PDH-E1 enzyme, which is inhibited and regulated by the PDH complex, consists of a hetero-tetramer containing two $\alpha$ subunits and two $\beta$ subunits. The E1- $\alpha$ subunit (PDH-E1 $\alpha$ ) contains the E1 active site and plays a key role in the function of the PDH complex (9). PDK activity has been reported in several types of carcinomas, such as colorectal cancer, bladder cancer, pancreatic cancer, and NSCLC (10-13). However, few studies have examined the relationship between $\mathrm{PDH}-\mathrm{E} 1 \alpha$ expression and clinicopathological factors associated with NSCLC. In this study, we aimed to determine whether PDH-E1 $\alpha$ may serve as a prognostic predictor for NSCLC after curative surgery.

We present the following article in accordance with the REMARK reporting checklist (available at https://dx.doi. org/10.21037/jtd-21-1463).

\section{Methods}

\section{Patient selection and ethical statement}

We retrospectively investigated the clinical course of 665 patients with NSCLC who underwent surgery at Osaka City University, Osaka, Japan, between January 2010 and December 2016. Patients with R1 or R2 surgery, with preoperative chemotherapy or radiation therapy, and patients without curative resection, such as wedge resection, segmentectomy, and lobectomy without mediastinal lymph node dissection, were excluded. A total of 445 patients with histologically confirmed primary NSCLC with pathological stage 0 to stage IIIA who underwent R0 surgery (more than lobectomy and mediastinal lymph node dissection) were enrolled in this study. Pathological findings were determined according to $8^{\text {th }}$ edition of the Union for International Cancer Control TNM classification. After final staging, the administration and regimen of adjuvant chemotherapy was appropriately determined by the cancer board consisting of a thoracic surgeon, a radiologist, and an oncologist. The study was conducted in accordance with the Declaration of Helsinki (as revised in 2013). This study was approved by the Osaka City University Ethics Committee (Reference number 2019-006). Informed consent was obtained from all patients. All methods with respect to humans performed in accordance with the relevant guidelines and regulations.

\section{Immunostaining of the PDH-E1a}

In brief, paraffin-embedded sections from 445 patients were deparaffinized in xylene and hydrated in decreasing concentrations of ethyl alcohol. The sections were incubated with $3 \%$ hydrogen peroxide to block endogenous peroxidase activity. The sections were then heated for $10 \mathrm{~min}$ at $105^{\circ} \mathrm{C}$ by autoclave in Target Retrieval Solution (DAKO, Carpinteria, CA, USA). Nonspecific binding was blocked via incubation with $10 \%$ normal rabbit serum for $10 \mathrm{~min}$. The specimens were incubated with anti PDHE1 $\alpha$ antibody (sc-377092; 1:100; Santa Cruz Biotechnology, Dallas, TX, USA; RRID:AB_2716767) (14) at $4{ }^{\circ} \mathrm{C}$ overnight. These sections were incubated with a mouse linker for $10 \mathrm{~min}$, and peroxidase-labeled polymer (Histofine SAB-PO(M) kit, Nichirei Biosciences Inc, Tokyo, Japan) for $5 \mathrm{~min}$, followed by counterstaining with Mayer's hematoxylin.

\section{Immunobistochemical determination}

The immunoreactivity of PDH-E $1 \alpha$ was evaluated according to the intensity of membranous staining at the deepest level of the tumor and the proportion of immunoreactive cells. Immunostaining intensity score was rated $0-3$ as follows: 0 , negative; $1+$, weakly positive; $2+$, positive; $3+$, strongly positive (Figure 1 ). The immunostaining proportion score was measured as an estimate of the proportion of positive cells: 0 , no 

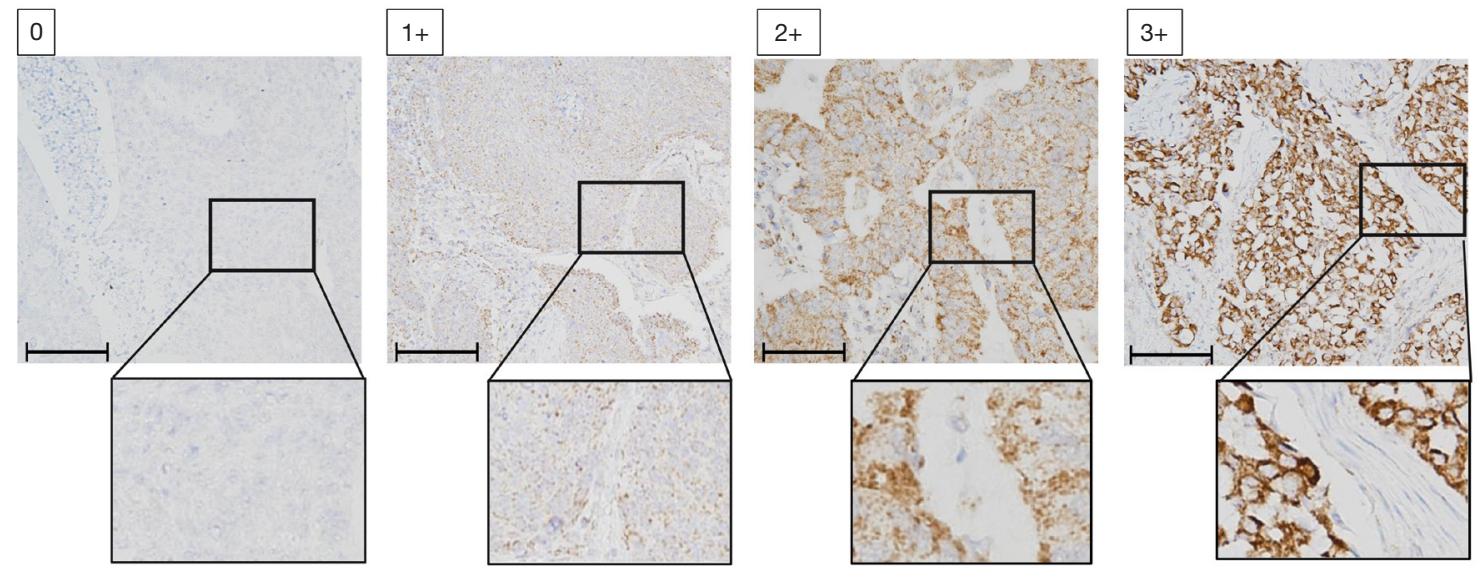

Figure 1 Representative images of immunostaining intensity of PDH-E1 $\alpha$ expression in patients with NSCLC. Intensity score: 0, negative; 1+, weakly positive; $2+$, positive; $3+$, strongly positive. Bar, $50 \mu \mathrm{m}$. PDH-E1 $\alpha$, pyruvate dehydrogenase E1 $\alpha$; NSCLC, non-small cell lung carcinoma.

immunoreactive cells; $1+,<30 \%$ immunoreactive cells; $2+$, $40-70 \%$ immunoreactive cells; $3+,>80 \%$ immunoreactive cells. The total score was calculated as the summation of the immunostaining intensity score and proportion score ranging from 0 to 6 , and $\mathrm{PDH}-\mathrm{E} 1 \alpha$ expression was considered positive when the summation score was $\geq 4$.

\section{Statistical analysis}

The $\chi^{2}$ test was performed to determine the significance of differences between covariates. Survival durations were calculated using the Kaplan-Meier method and analyzed using the log-rank test to compare the cumulative survival durations in the patient groups. In addition, the Cox proportional hazards model was used to compute the multivariate hazard ratios for the study parameters. In all tests, a $\mathrm{P}$ value $<0.05$ was considered significant. All statistical analyses were performed using EZR (Saitama Medical Center, Jichi Medical University, Saitama, Japan), which is a graphical user interface of $\mathrm{R}$, and more precisely, a modified version of the $\mathrm{R}$ commander (The $\mathrm{R}$ Foundation for Statistical Computing, Vienna, Austria) (15).

\section{Results}

\section{Relationship between PDH-E1 1 expression and clinicopathological features}

The clinicopathological features of all 445 patients based on PDH-E1 $\alpha$ expression are summarized in Table 1. In total, 248 patients (56\%) of the 445 patients with NSCLC patients were $\mathrm{PDH}-\mathrm{E} 1 \alpha$-positive and 197 patients were PDH-E1 $\alpha$-negative. PDH-E1 $\alpha$ positivity was significantly correlated with the presence of adenocarcinoma $(\mathrm{P}<0.001)$ in comparison to the $\mathrm{PDH}-\mathrm{E} 1 \alpha$-negative group. No other clinicopathological features were correlated with PDH-E1 $\alpha$ expression, including induction of adjuvant chemotherapy and postoperative complications. There was no significant correlation between PDH-E1 $\alpha$ expression and clinicopathological features in patients with adenocarcinoma or with squamous cell carcinoma, respectively.

\section{Correlation between PDH-E1a expression and survival of patients with NSCLC}

The 5-year overall survival rate according to PDH-E1 $\alpha$ expression in all 445 patients is presented in Figure 2. Patients with NSCLC showing PDH-E1 $\alpha$-negative expression had significantly poorer survival rates $(\mathrm{P}=0.007)$ than those of patients showing PDH-E1 $\alpha$-positive expression. According to the pathological stages, the 5 -year overall survival rate of patients with NSCLC showing PDH-E1 $\alpha$-negative expression was a significantly poorer $(\mathrm{P}=0.026)$ than those showing PDH-E1 $\alpha$-positive expression in stage II. In contrast, no significant difference was found between $\mathrm{PDH}-$ E1 $\alpha$ expression in stages $0-\mathrm{I}$ and III.

The 5-year disease-free survival rate, according to $\mathrm{PDH}-\mathrm{E} 1 \alpha$ expression in all 445 patients, is presented in Figure 3. Patients with NSCLC showing PDH-E1 $\alpha$ negative expression had significantly poorer survival 
Table 1 Relationship between expression of PDH-E1 $\alpha$ and clinicopathological features in 445 patients with NSCLC

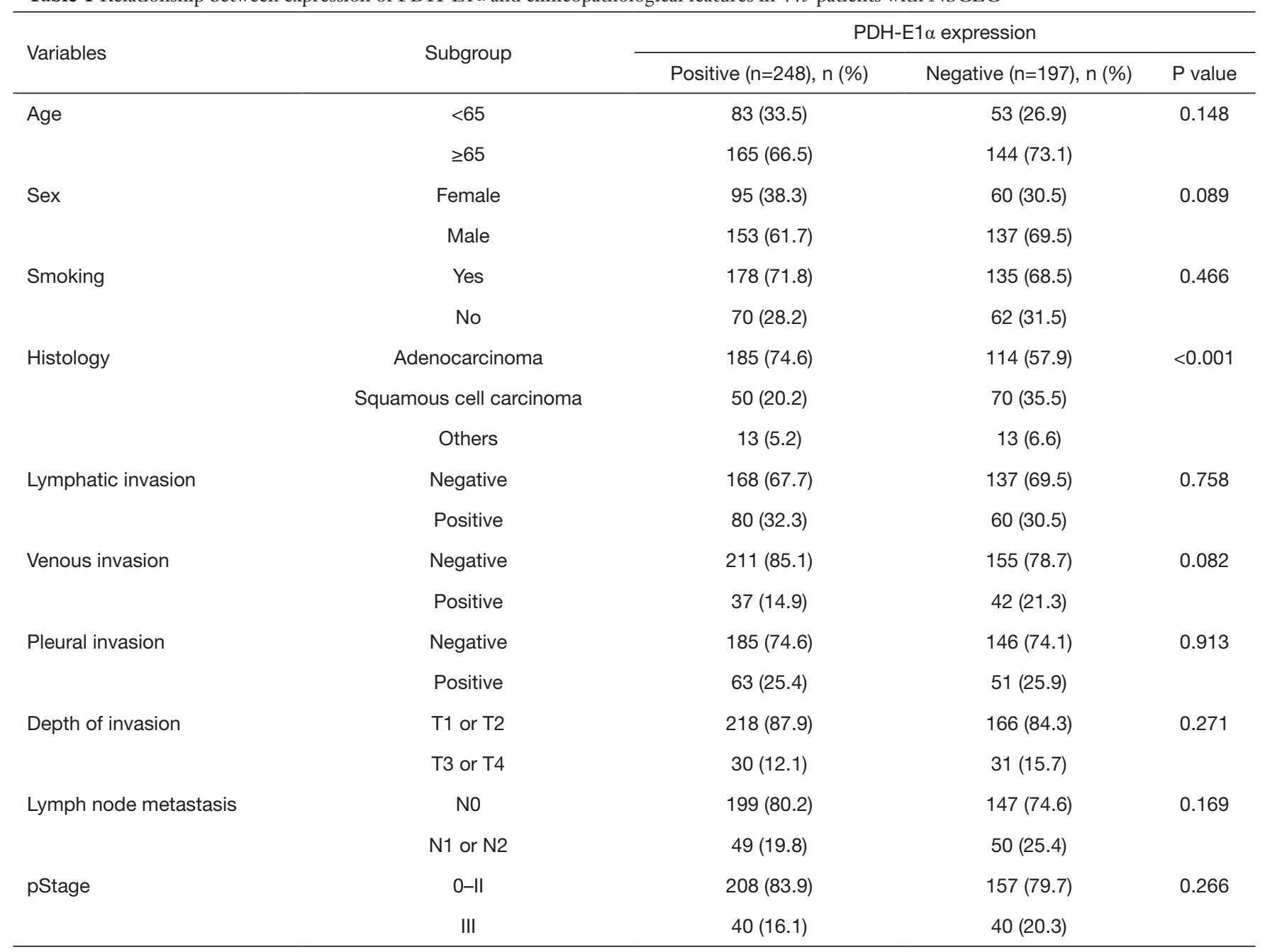

PDH-E1 $\alpha$, pyruvate dehydrogenase E1 $\alpha$; NSCLC, non-small cell lung carcinoma.

rates $(\mathrm{P}=0.02)$ than patients showing $\mathrm{PDH}-\mathrm{E} 1 \alpha$-positive expression. According to the pathological stages, no significant difference was found between PDH-E1 $\alpha$ expression in all stages.

There was no significant correlation between PDH$\mathrm{E} 1 \alpha$ expression and overall and disease-free survival rates in patients with adenocarcinoma or squamous cell carcinoma, respectively.

\section{Univariate and multivariate analyses of survival}

The results of the univariate and multivariate analyses of the overall survival are presented in Table 2. Univariate analysis showed that poor overall survival was significantly correlated with PDH-E1 $\alpha$ negativity $(\mathrm{P}=0.007)$, male sex
( $\mathrm{P}<0.001)$, smoking $(\mathrm{P}=0.049)$, histology of adenocarcinoma $(\mathrm{P}=0.001)$, lymphatic invasion $(\mathrm{P}=0.006)$, pleural invasion $(\mathrm{P}=0.002)$, pathological T3/4 $(\mathrm{P}=0.02)$, and lymph node metastasis $(\mathrm{P}=0.014)$. Multivariate analysis revealed that PDH-E1 $\alpha$ negativity $(\mathrm{P}=0.037)$ and male sex $(\mathrm{P}<0.001)$ were significantly correlated with poor overall survival.

Table 3 shows the univariate and multivariate analyses results of the disease-free survival. Univariate analysis showed that a poor disease-free survival was significantly correlated with PDH-E1 $\alpha$ negativity $(\mathrm{P}=0.021)$, male sex $(\mathrm{P}<0.001)$, histology of adenocarcinoma $(\mathrm{P}=0.029)$, venous invasion $(\mathrm{P}<0.001)$, lymphatic invasion $(\mathrm{P}<0.001)$, pleural invasion $(\mathrm{P}<0.001)$, pathological T3/4 $(\mathrm{P}<0.001)$, lymph node metastasis $(\mathrm{P}<0.001)$, and adjuvant chemotherapy $(\mathrm{P}=0.005)$. Multivariate analysis revealed that male sex 

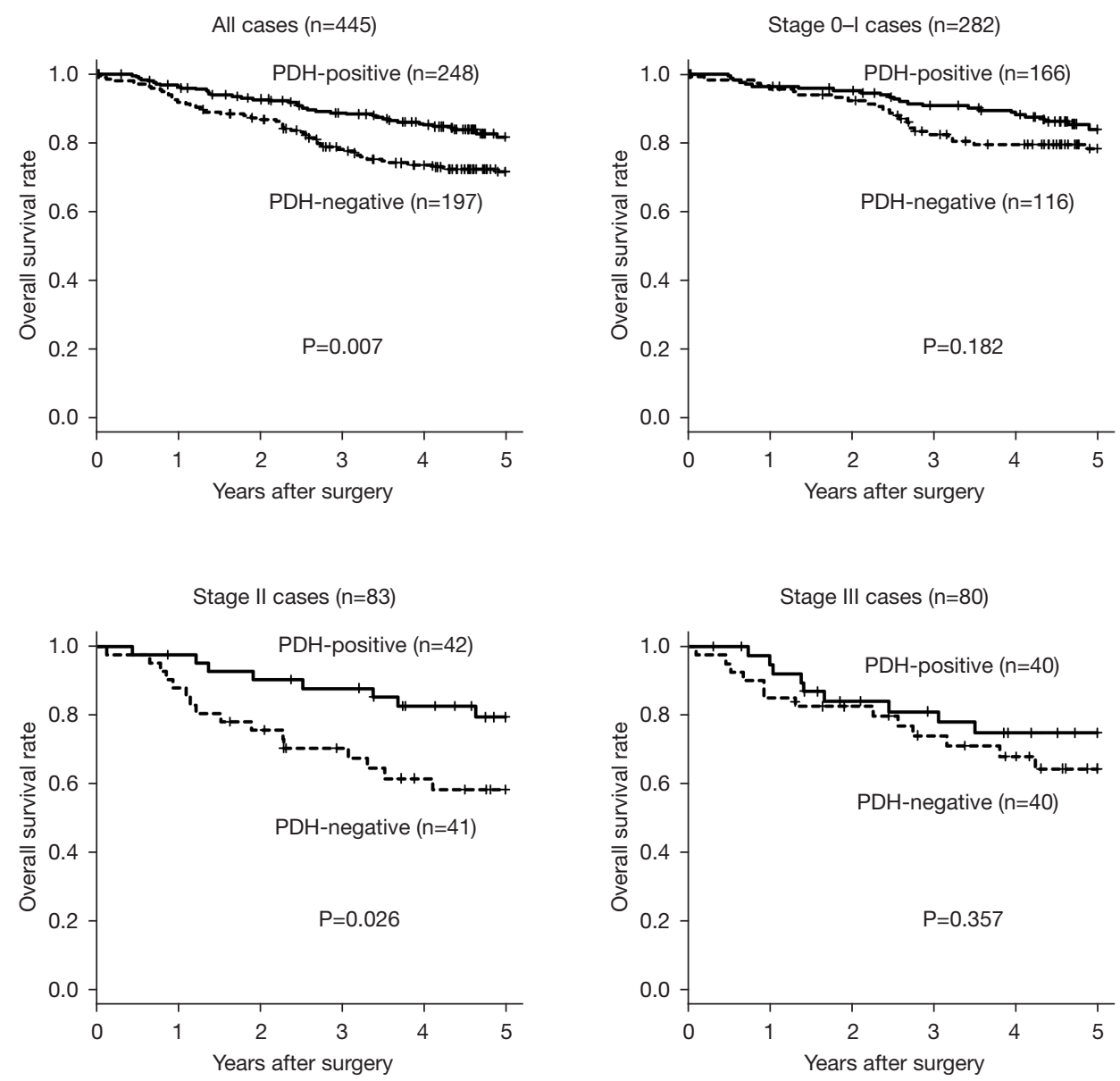

Figure 2 Relationship between overall survival rate and PDH-E1 $\alpha$ expression. The 5 -year overall survival rate in 445 patients with NSCLC. Patients showing negative PDH-E1 $\alpha$ expression have significantly poorer survival rates $(\mathrm{P}=0.007)$ than those showing positive $\mathrm{PDH}-\mathrm{E} 1 \alpha$ expression. The PDH-E1 $\alpha$ expression in associated with the pathological stage was evaluated. A significant difference in the association of survival rate and $\mathrm{PDH}-\mathrm{E} 1 \alpha$ expression in pathological stage II $(\mathrm{P}=0.026)$ was observed, but not for stages $0-\mathrm{I}$ and III. PDH-E1 $\alpha$, pyruvate dehydrogenase E1 $\alpha$; NSCLC, non-small cell lung carcinoma.

$(\mathrm{P}=0.002)$, pleural invasion $(\mathrm{P}=0.002)$, and lymph node metastasis $(\mathrm{P}<0.001)$ were significantly correlated with a poor disease-free survival, while $\mathrm{PDH}-\mathrm{E} 1 \alpha$ negativity was $\operatorname{not}(\mathrm{P}=0.068)$.

\section{Discussion}

Lung cancer is associated with a high morbidity and mortality rate worldwide; however, no reliable and independent prognostic predictor for NSCLC after curative surgery is available. PDH activity has been found to be associated with various cancers. However, few studies have evaluated the relationship between PDH-E1 $\alpha$ expression and clinicopathological factors associated with NSCLC. In this study, PDH-E1 $\alpha$ expression in NSCLC was found to be significantly correlated with the presence of adenocarcinoma. It has been reported that histological adenocarcinoma type is associated with a higher oxygen-containing environment than that of squamous cell-type carcinoma in NSCLC (16). Normal cells obtain energy from glucose via mitochondrial oxidative phosphorylation under aerobic conditions (17). These findings suggest that the expression level of PDHE1 $\alpha$ might have been higher in adenocarcinoma than in squamous cell carcinoma due to the relatively higher oxygen levels in the adenocarcinoma environment.

A cell produces 34 molecules of adenosine triphosphate (ATP) from a single molecule of glucose through the glycolysis to TCA cycle and the electron transfer 

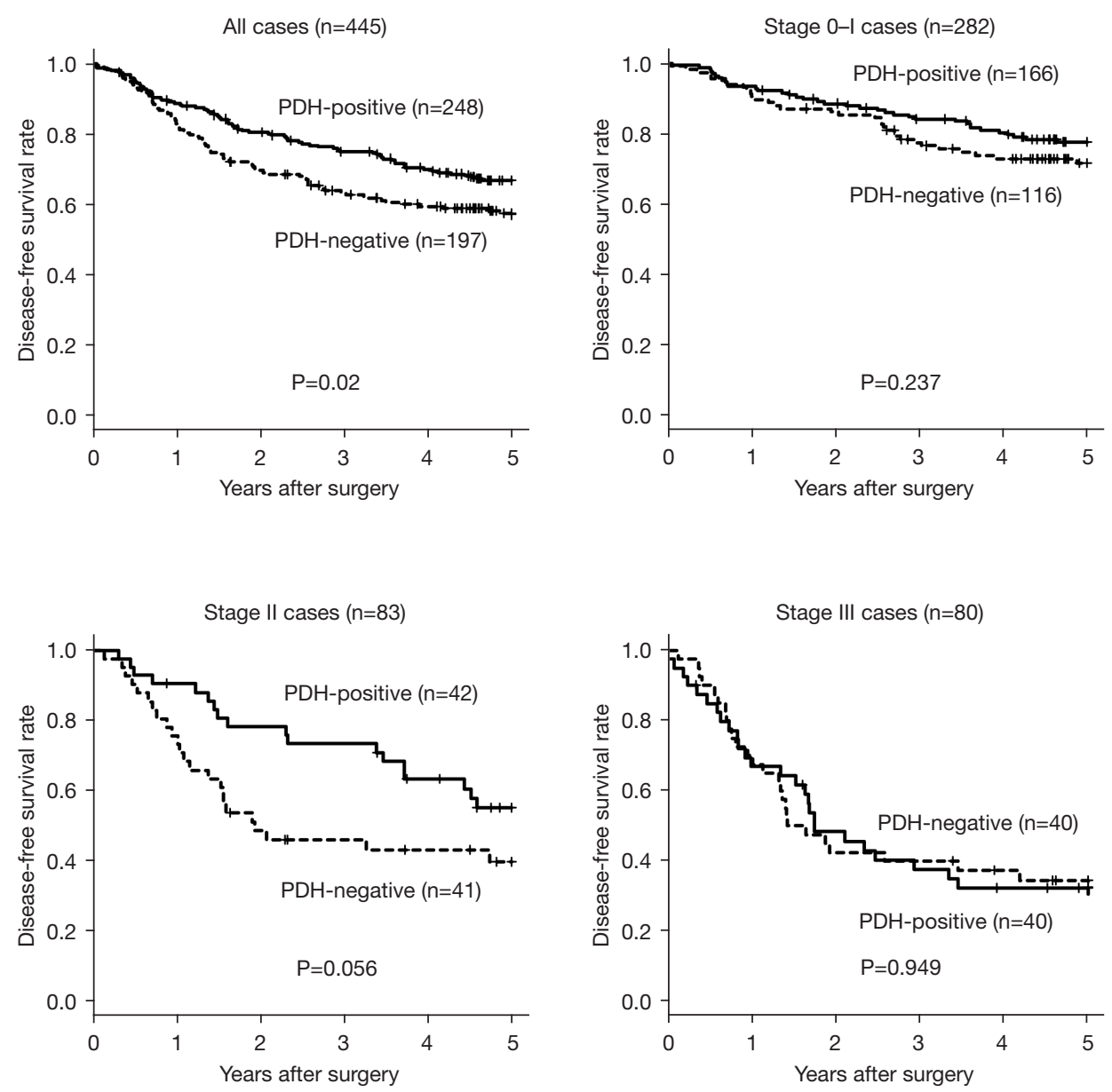

Figure 3 Relationship between disease-free survival rate and PDH-E1 $\alpha$ expression. 5-year disease-free survival rate in 445 patients with NSCLC. Patients showing negative PDH expression had significantly poorer survival rates $(\mathrm{P}=0.02)$ than those showing positive $\mathrm{PDH}$ expression. No significant difference was found in the survival rate according to the $\mathrm{PDH}$ expression associated with pathological stage. PDH-E1 $\alpha$, pyruvate dehydrogenase E1 $\alpha$; NSCLC, non-small cell lung carcinoma.

system under normoxic conditions, called oxidative phosphorylation. On the other hand, a single molecule of glucose provides two molecules of ATP in a hypoxic environment, called anaerobic glycolysis. These two different metabolic pathways diverge at pyruvate. PDHE1 $\alpha$ catalyzes the conversion of pyruvate to acetyl-CoA, which enters into the TCA cycle, and PDH-E1 $\alpha$ negativity induces anaerobic glycolysis. As anaerobic glycolysis is upregulated in cancer cells, extracellular acidosis is caused by the accumulation of lactate (18). Normal cells show a low tolerance to acidosis; however, cancer cells can readily adapt to acidosis via mutations such as in $\mathrm{p} 53$, and thereby continue to proliferate (19). Furthermore, when metastatic cancer cells proliferate by occluding the intravascular space, the tolerance to hypoxic environments and acidosis is advantageous to cancer cells (20). These findings suggest that anaerobic glycolysis may be upregulated and PDHE1 $\alpha$ expression may be downregulated in cancer cells with malignancy potential.

Patients with NSCLC showing PDH-E1 $\alpha$ expression were found to have significantly higher overall survival rates than those showing $\mathrm{PDH}-\mathrm{E} 1 \alpha$-negative expression in pathological stage II. PDH-E1 $\alpha$ expression may serve as an independent prognostic factor in patients with NSCLC treated with R0 resection, and was not significantly correlated with postoperative complications or administration of adjuvant chemotherapy. Low PDH-E1 $\alpha$ levels in cancer cells are closely related to the malignancy potential of cancer cells, such as distant metastasis (20), suggesting that $\mathrm{PDH}-\mathrm{E} 1 \alpha$ may represent a reliable 
Table 2 Univariate and multivariate analysis of 5 -year overall survival of 445 patients with NSCLC

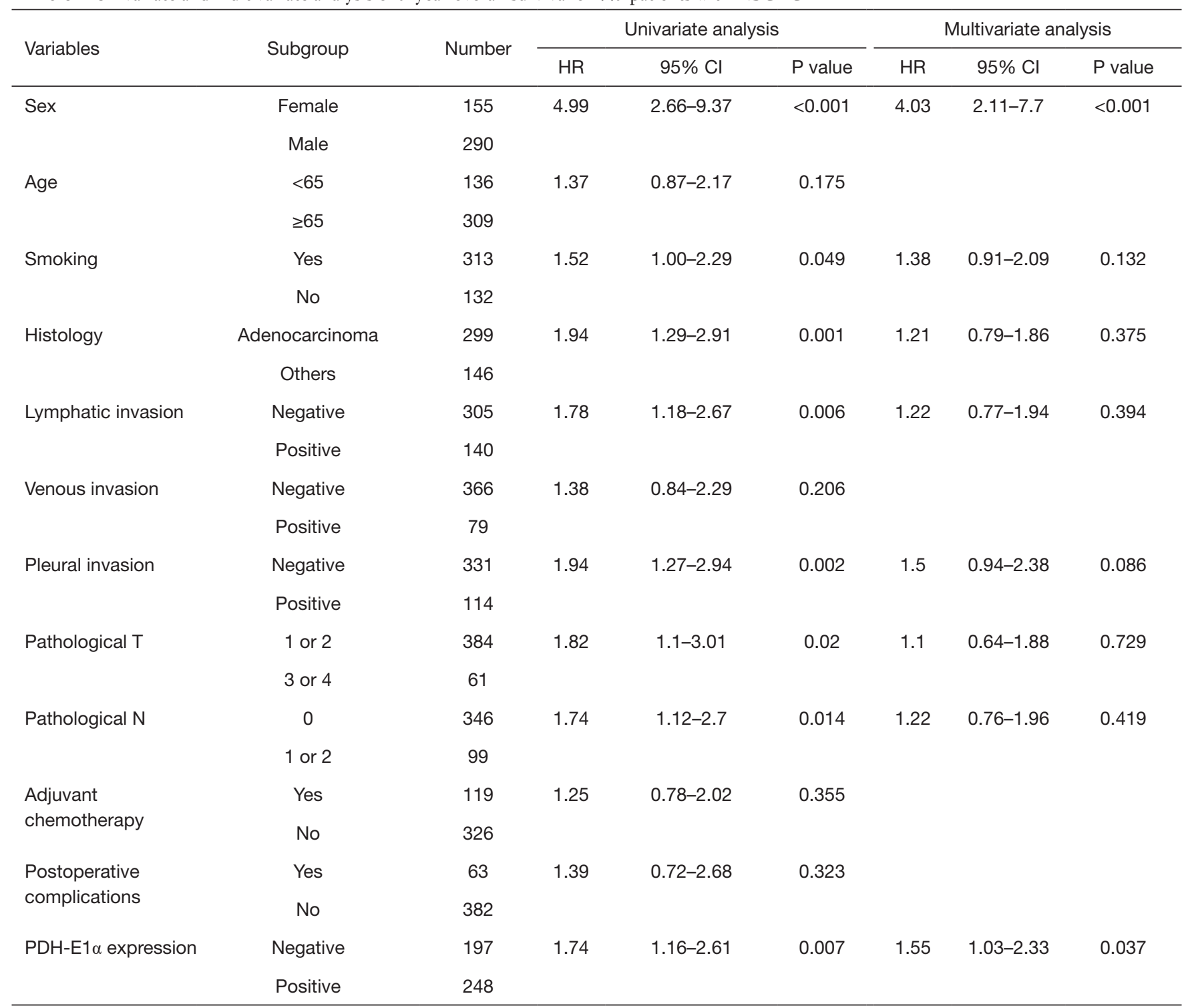

NSCLC, non-small cell lung carcinoma; Cl, confidence interval; HR, hazard ratio; PDH-E1 $\alpha$, pyruvate dehydrogenase E1 $\alpha$.

prognostic predictor for patients with NSCLC after curative operation, especially at stage II.

However, PDH-E1 $\alpha$-negative expression had a significantly poorer survival rate in pathological stage II only, not in stages $0-\mathrm{I}$ and III. This is probably because the expression of PDH-E1 $\alpha$ indicates the potential of cancer malignancy. In patients with pathological stage 0 I NSCLC, the good effect of surgery for prognosis may be larger than the malignant potential of PDH negativity. On the other hand, the prognosis of patients with pathological stage IIIA NSCLC may have been significantly affected by cancer progression rather than by the malignant potential of PDH-E1 $\alpha$ negativity.

To the best of our knowledge, this is the first report to shows that $\mathrm{PDH}-\mathrm{E} 1 \alpha$ represents a reliable prognostic predictor for patients with NSCLC resected via curative operation. These findings suggest that PDH-E1 $\alpha$ plays an important role in NSCLC development. PDH-E1 $\alpha$ or PDK may serve as potential target in the treatment of NSCLC (21-23). Further investigation on the regulation of PDH-E1 $\alpha$ expression may assist in identifying a promising therapeutic target for effective treating stage II NSCLC. 
Table 3 Univariate and multivariate analysis of 5 -year disease-free survival of 445 patients with NSCLC

\begin{tabular}{|c|c|c|c|c|c|c|c|c|}
\hline Variables & Subgroup & Number & \multicolumn{3}{|c|}{ Univariate analysis } & \multicolumn{3}{|c|}{ Multivariate analysis } \\
\hline \multirow[t]{2}{*}{ Sex } & Female & 155 & 2.42 & $1.66-3.54$ & $<0.001$ & 1.86 & $1.25-2.76$ & 0.002 \\
\hline & Male & 290 & & & & & & \\
\hline Age & $<65$ & 136 & 1.29 & $0.92-1.83$ & 0.144 & & & \\
\hline \multirow[t]{2}{*}{ Smoking } & Yes & 313 & 1.29 & $0.93-1.78$ & 0.121 & & & \\
\hline & No & 132 & & & & & & \\
\hline \multirow[t]{2}{*}{ Histology } & Adenocarcinoma & 299 & 1.42 & $1.04-1.95$ & 0.029 & 0.84 & $0.59-1.19$ & 0.318 \\
\hline & Others & 146 & & & & & & \\
\hline \multirow[t]{2}{*}{ Venous invasion } & Negative & 366 & 2.28 & $1.61-3.22$ & $<0.001$ & 1.21 & $0.83-1.77$ & 0.332 \\
\hline & Positive & 79 & & & & & & \\
\hline \multirow[t]{2}{*}{ Pleural invasion } & Negative & 331 & 2.53 & $1.84-3.46$ & $<0.001$ & 1.77 & $1.23-2.55$ & 0.002 \\
\hline & Positive & 114 & & & & & & \\
\hline \multirow[t]{2}{*}{ Pathological T } & 1 or 2 & 384 & 2.31 & $1.6-3.34$ & $<0.001$ & 1.45 & $0.95-2.2$ & 0.087 \\
\hline & 3 or 4 & 61 & & & & & & \\
\hline \multirow[t]{2}{*}{ Pathological N } & 0 & 346 & 3.1 & $2.26-4.27$ & $<0.001$ & 2.42 & $1.66-3.53$ & $<0.001$ \\
\hline & 1 or 2 & 99 & & & & & & \\
\hline PDH-E1 $\alpha$ expression & Positive & 248 & & & & & & \\
\hline
\end{tabular}

NSCLC, non-small cell lung carcinoma; Cl, confidence interval; HR, hazard ratio; PDH-E1 $\alpha$, pyruvate dehydrogenase E1 $\alpha$.

This study has some limitations. First, this is a retrospective study. It was not possible to analyze all factors in all patients, and some patients dropped out of the trial and could not be followed up upon. Second, we evaluated PDH-E1 $\alpha$ expression only, but did not include all PDH subunits such as E1 $\beta, \mathrm{E} 2$, or E3. In addition, PDH-E1 $\alpha$ expression was evaluated only by immunohistochemistry. The accumulation of gene or mRNA data would be ideal for supporting the results of this study.
In conclusion, $\mathrm{PDH}-\mathrm{E} 1 \alpha$ represents a reliable prognostic predictor for patients with NSCLC undergoing curative resection. PDK may serve as a promising target for patients with NSCLC.

\section{Acknowledgments}

Funding: This study was supported by KAKENHI (Grantin-Aid for Scientific Research) [21H03008]. 


\section{Footnote}

Reporting Checklist: The authors have completed the REMARK reporting checklist. Available at https://dx.doi. org/10.21037/jtd-21-1463

Data Sharing Statement: Available at https://dx.doi. org/10.21037/jtd-21-1463

Peer Review File: Available at https://dx.doi.org/10.21037/ jtd-21-1463

Conflicts of Interest: All authors have completed the ICMJE uniform disclosure form (available at https://dx.doi. org/10.21037/jtd-21-1463). The authors have no conflicts of interest to declare.

Ethical statement: The authors are accountable for all aspects of the work in ensuring that questions related to the accuracy or integrity of any part of the work are appropriately investigated and resolved. The study was conducted in accordance with the Declaration of Helsinki (as revised in 2013). The study was approved by the Osaka City University Ethics Committee (Reference number 2019006) and informed consent was obtained from all individual participants.

Open Access Statement: This is an Open Access article distributed in accordance with the Creative Commons Attribution-NonCommercial-NoDerivs 4.0 International License (CC BY-NC-ND 4.0), which permits the noncommercial replication and distribution of the article with the strict proviso that no changes or edits are made and the original work is properly cited (including links to both the formal publication through the relevant DOI and the license). See: https://creativecommons.org/licenses/by-nc-nd/4.0/.

\section{References}

1. Torre LA, Bray F, Siegel RL, et al. Global cancer statistics, 2012. CA Cancer J Clin 2015;65:87-108.

2. Maemondo M, Inoue A, Kobayashi K, et al. Gefitinib or chemotherapy for non-small-cell lung cancer with mutated EGFR. N Engl J Med 2010;362:2380-8.

3. Molina JR, Yang P, Cassivi SD, et al. Non-small cell lung cancer: epidemiology, risk factors, treatment, and survivorship. Mayo Clin Proc 2008;83:584-94.

4. Abdel-Wahab AF, Mahmoud W, Al-Harizy RM. Targeting glucose metabolism to suppress cancer progression: prospective of anti-glycolytic cancer therapy. Pharmacol Res 2019;150:104511.

5. Lebelo MT, Joubert AM, Visagie MH. Warburg effect and its role in tumourigenesis. Arch Pharm Res 2019;42:833-47.

6. WARBURG O. On the origin of cancer cells. Science 1956;123:309-14.

7. Thews $\mathrm{O}$, Riemann A. Tumor $\mathrm{pH}$ and metastasis: a malignant process beyond hypoxia. Cancer Metastasis Rev 2019;38:113-29.

8. Pavlova NN, Thompson CB. The Emerging Hallmarks of Cancer Metabolism. Cell Metab 2016;23:27-47.

9. Ozden O, Park SH, Wagner BA, et al. SIRT3 deacetylates and increases pyruvate dehydrogenase activity in cancer cells. Free Radic Biol Med 2014;76:163-72.

10. Yang Z, Zhang SL, Hu X, et al. Inhibition of pyruvate dehydrogenase kinase 1 enhances the anti-cancer effect of EGFR tyrosine kinase inhibitors in non-small cell lung cancer. Eur J Pharmacol 2018;838:41-52.

11. Jin L, Kim EY, Chung TW, et al. Hemistepsin A suppresses colorectal cancer growth through inhibiting pyruvate dehydrogenase kinase activity. Sci Rep 2020;10:21940.

12. Woolbright BL, Choudhary D, Mikhalyuk A, et al. The Role of Pyruvate Dehydrogenase Kinase-4 (PDK4) in Bladder Cancer and Chemoresistance. Mol Cancer Ther 2018;17:2004-12.

13. Tambe Y, Terado T, Kim CJ, et al. Antitumor activity of potent pyruvate dehydrogenase kinase 4 inhibitors from plants in pancreatic cancer. Mol Carcinog 2019;58:1726-37.

14. Yang Z, Wang Y, Zhang L, et al. Phosphorylated form of pyruvate dehydrogenase $\alpha 1$ mediates tumor necrosis factor $\alpha$-induced glioma cell migration. Oncol Lett 2021;21:176.

15. Kanda Y. Investigation of the freely available easy-touse software 'EZR' for medical statistics. Bone Marrow Transplant 2013;48:452-8.

16. Schuurbiers OC, Meijer TW, Kaanders JH, et al. Glucose metabolism in NSCLC is histology-specific and diverges the prognostic potential of 18FDG-PET for adenocarcinoma and squamous cell carcinoma. J Thorac Oncol 2014;9:1485-93.

17. Li Z, Zhang H. Reprogramming of glucose, fatty acid and amino acid metabolism for cancer progression. Cell Mol Life Sci 2016;73:377-92.

18. de la Cruz-López KG, Castro-Muñoz LJ, ReyesHernández DO, et al. Lactate in the Regulation of Tumor 
Microenvironment and Therapeutic Approaches. Front Oncol 2019;9:1143.

19. Lamonte G, Tang X, Chen JL, et al. Acidosis induces reprogramming of cellular metabolism to mitigate oxidative stress. Cancer Metab 2013;1:23.

20. Gatenby RA, Gillies RJ. Why do cancers have high aerobic glycolysis? Nat Rev Cancer 2004;4:891-9.

21. Anwar S, Mohammad T, Shamsi A, et al. Discovery of Hordenine as a Potential Inhibitor of Pyruvate Dehydrogenase Kinase 3: Implication in Lung Cancer

Cite this article as: Ito R, Yashiro $M$, Tsukioka T, Izumi N, Komatsu H, Inoue H, Yamamoto Y, Nishiyama N. Pyruvate dehydrogenase $\mathrm{E} 1 \alpha$ represents a reliable prognostic predictor for patients with non-small cell lung cancer resected via curative operation. J Thorac Dis 2021;13(10):5691-5700. doi: 10.21037/ jtd-21-1463
Therapy. Biomedicines 2020;8:119.

22. Lee EJ, Chung TW, Lee JH, et al. Water-extracted branch of Cinnamomum cassia promotes lung cancer cell apoptosis by inhibiting pyruvate dehydrogenase kinase activity. J Pharmacol Sci 2018;138:146-54.

23. Guo F, Zhao S, Li X. Discovery of novel pyruvate dehydrogenase kinases inhibitors by screening of an in-house small molecule library for anti-lung cancer therapeutics. Bioorg Med Chem Lett 2019;29:291-6. 